\title{
Dispersal patterns and population genetic structure of Aedes albopictus (Diptera: Culicidae) in three different climatic regions of China
}

Jian Gao ${ }^{\dagger}$, Heng-Duan Zhang ${ }^{\dagger}$, Xiao-Xia Guo, Dan Xing, Yan-De Dong, Ce-Jie Lan, Ge Wang, Chao-Jie Li, Chun-Xiao Li and Tong-Yan Zhao*

\begin{abstract}
Background: Aedes albopictus is an indigenous primary vector for dengue and Zika viruses in China. Compared with its insecticide resistance, biology and vector competence, little is known about its genetic variation, which corresponds to environmental variations. Thus, the present study examines how Ae. albopictus varies among different climatic regions in China and deciphers its potential dispersal patterns.

Methods: The genetic variation and population structure of 17 Ae. albopictus populations collected from three climatic regions of China were investigated with 11 microsatellite loci and the mitochondrial coxl gene.

Results: Of 44 isolated microsatellite markers, 11 pairs were chosen for genotyping analysis and had an average PIC value of 0.713 , representing high polymorphism. The number of alleles was high in each population, with the $n_{e}$ value increasing from the temperate region (3.876) to the tropical region (4.144). Twenty-five cox/ haplotypes were detected, and the highest diversity was observed in the tropical region. The mean Ho value (ca. 0.557 ) of all the regions was significantly lower than the mean He value (ca. 0.684), with nearly all populations significantly departing from HWE and displaying significant population expansion ( $p$ value $<0.05$ ). Two genetically isolated groups and three haplotype clades were evaluated via STRUCTURE and haplotype phylogenetic analyses, and the tropical populations were significantly isolated from those in the other regions. Most genetic variation in Ae. albopictus was detected within populations and individuals at 31.40 and $63.04 \%$, respectively, via the AMOVA test, and a relatively significant positive correlation was observed among only the temperate populations via IBD analysis $\left(R^{2}=0.6614, p=0.048\right)$. Recent dispersions were observed among different $A$ e. albopictus populations, and four major migration trends with high gene flow $(\mathrm{Nm}>0.4)$ were reconstructed between the tropical region and the other two regions. Environmental factors, especially temperature and rainfall, may be the leading causes of genetic diversity in different climatic regions.
\end{abstract}

Conclusions: Continuous dispersion contributes to the genetic communication of Ae. albopictus populations across different climatic regions, and environmental factors, especially temperature and rainfall, may be the leading causes of genetic variation.

\footnotetext{
*Correspondence: aedes@126.com; tongyanzhao@126.com

†ian Gao and Heng-Duan Zhang contributed equally to this work State Key Laboratory of Pathogen and Biosecurity, Beijing Institute of Microbiology and Epidemiology, Beijing 100071, China
}

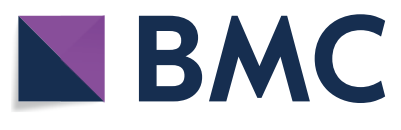

(c) The Author(s) 2021. This article is licensed under a Creative Commons Attribution 4.0 International License, which permits use, sharing, adaptation, distribution and reproduction in any medium or format, as long as you give appropriate credit to the original author(s) and the source, provide a link to the Creative Commons licence, and indicate if changes were made. The images or other third party material in this article are included in the article's Creative Commons licence, unless indicated otherwise in a credit line to the material. If material is not included in the article's Creative Commons licence and your intended use is not permitted by statutory regulation or exceeds the permitted use, you will need to obtain permission directly from the copyright holder. To view a copy of this licence, visit http://creativecommons.org/licenses/by/4.0/. The Creative Commons Public Domain Dedication waiver (http://creativecommons.org/publicdomain/zero/1.0/) applies to the data made available in this article, unless otherwise stated in a credit line to the data. 
Keywords: Aedes albopictus, Genetic variation, Haplotype, Dispersion pattern, Climatic regions, Microsatellite loci, Environmental factors

\section{Background}

Aedes (Stegomyia) albopictus, also known as the Asian tiger mosquito, is an epidemiologically critical vector for its transmission of several arboviruses, including dengue virus (DENV), Zika virus (ZIKV), chikungunya virus and filarial nematodes, such as Dirofilaria immitis [1-4]. Compared with Aedes aegypti, the primary vector for DENV and ZIKV, Ae. albopictus is generally considered to be a less efficient vector due to its less anthropophilic behavior and inferior adaptation to urban domestic environments [5]. However, its broad temperature adaptability and high competence for several arboviruses make it approximately as important a vector as Ae. aegypti. Meanwhile, combined with its strong ecological plasticity, global trade, such as the transportation of used tires and "lucky bamboo," has also accelerated the global spread of this species from its original Southeastern Asian distribution to every continent except Antarctica [6]. Currently, it is among the top 100 worst invasive species and a threat to human health worldwide [7].

Natural environmental variation is responsible for fluctuations in insect population dynamics, distribution and biology, including population number, intensity and feeding behavior [8]. An important and rapidly adapting insect with high fecundity and a short life cycle, Ae. albopictus has population dynamics and vector competence that are also greatly influenced by environmental conditions $[9,10]$. Numerous previous studies have discussed the effects of environmental variations on $A e$. albopictus populations, mainly based on their influence on mosquito abundance, survival, size, fecundity and competence for certain arboviruses [11-15]. However, less research has focused on changes in the genetic diversity and population structure of $A e$. albopictus populations associated with various environmental conditions [16-18], although this information is essential for reconstructing the dispersion patterns of Ae. albopictus in certain regions and providing necessary information for subsequent mosquito control.

With the advent of urbanization in China, the natural environment was altered tremendously; more people have moved from rural areas to cities, and trade has increased between cities and rural areas. All these processes create suitable habitats (e.g. waste tires, vases, plastic pails and concrete tanks) for Ae. albopictus and facilitate its breeding and dispersal throughout the country [19-22]. Meanwhile, the dispersion of Ae. albopictus from its original environment to a new environment facilitates the manifestation of new phenotypes and the increase of mutated alleles in frequency, and thriving international travel and trade provide essential routes for the continuous introduction of arboviruses from other countries, especially those in Southeast Asia, where Ae. albopictus originated [23], thus putting people at risk worldwide. During the past 10 years, at least 22 imported ZIKV cases were confirmed by the China $\mathrm{CDC}$, and the persistent emergence of dengue in Southern China, especially in Guangdong and Yunnan provinces, is positively correlated to the widespread presence of Ae. aegypti and Ae. albopictus among these regions [24]. In contrast to Ae. aegypti (an invasive species distributed only in the southern area of China), Ae. albopictus is an indigenous Chinese mosquito, ranging from Dalian in the north to Hainan in the south $[25,26]$. Moreover, it has been considered the sole agent of numerous recent dengue fever outbreaks in China [27, 28]. Its wide distribution and high competence for numerous arboviruses and nematode parasites emphasize the need to more extensively study the biology, distribution and dispersion patterns of this species.

High genetic variation is ubiquitous in vector populations, especially in invasive vectors, such as Ae. aegypti and Ae. albopictus, where it helps them occupy diverse niches and respond quickly to evolutionary challenges [29]. Microsatellites are the preferred markers for studying genetic variation in vectors due to their codominance, high informativity and vast abundance throughout vector genomes [30]. To date, multiple microsatellite loci associated with Ae. albopictus have been successfully isolated and employed in Ae. albopictus population studies on a global scale, and they continue to be a popular choice of genetic marker [15, 31-33]. Simultaneously and independently from Ae. albopictus microsatellites, the mitochondrial coxI gene has frequently been used for mosquito barcoding and invasive species monitoring because of its conservation and high accuracy when distinguishing sequence variation at the interspecies level and its usefulness in investigating population dispersal routes of mosquitoes [34-38].

To evaluate how environmental factors affect genetic diversity and dispersal patterns of Ae. albopictus and decipher the potential dispersal patterns of Ae. albopictus among different climatic regions, a total of $17 \mathrm{Ae}$. albopictus populations were collected and examined via 
11 microsatellite loci and the coxI gene. The results provide information for the future control of Ae. albopictus in China.

\section{Materials and methods}

Eco-climatic characteristics of different climatic regions

All mosquitoes were sampled from the three main climatic regions of China, including tropical, subtropical and temperate regions. The annual accumulated temperature and precipitation for the tropical region is > $8000{ }^{\circ} \mathrm{C}$ and $1500-2000 \mathrm{~mm}$, respectively, with the lowest monthly average temperature being $15-24{ }^{\circ} \mathrm{C}$. These data for the subtropical region are between 4500 and $8000{ }^{\circ} \mathrm{C}$ and $>1000 \mathrm{~mm}$, respectively, with the lowest monthly average temperature being $0-15{ }^{\circ} \mathrm{C}$. These data for the temperate region are between 3000 and $4500{ }^{\circ} \mathrm{C}$ and $700 \mathrm{~mm}$, respectively, with the lowest monthly average temperature being -8 to $0{ }^{\circ} \mathrm{C}[39,40]$.

\section{Mosquito sampling and DNA isolation}

In the present study, ca. 600 Ae. albopictus larvae were sampled from 17 geographically diverse sites across three environmentally distinct regions of China from June to August 2018; all the sampling site information is described in Fig. 1 and Additional file 1: Table S1. To avoid inbreeding interference, each pool of larval mosquitoes for a given locality was collected from at least five wild breeding places within $500 \mathrm{~m}$. For each of the samples, the larvae were reared separately and emerged at $25 \pm 1{ }^{\circ} \mathrm{C}$ at $75 \pm 5 \%$ relative humidity (RH) under a $14 \mathrm{~h}$ light $/ 10 \mathrm{~h}$ dark (LD) photoperiod, and all female adult mosquitoes were identified under a microscope before DNA isolation [41, 42]. The DNA isolation was conducted with the Qiagen DNeasy Blood and Tissue Kit (no. 69504, Qiagen, Germany) according to the manufacturer's protocol, and all the DNA samples were stored at $-80^{\circ} \mathrm{C}$.

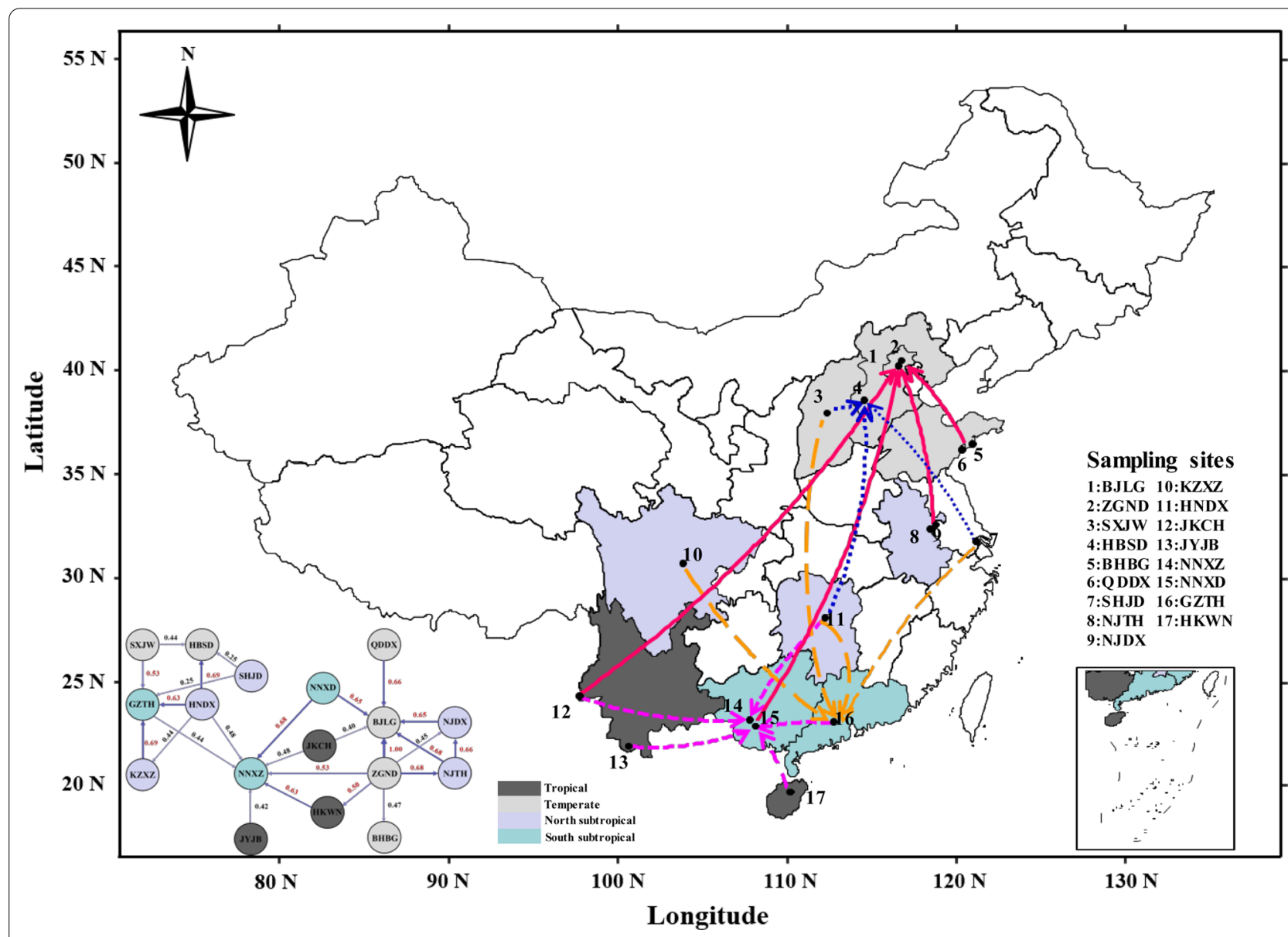

Fig. 1 Sampling map and migration patterns for Ae. albopictus in three different climatic regions of China. Three climatic regions were marked with different colors, four major destinations were marked with stars of different colors, and migration routes were marked with different types of arrows 


\section{Microsatellite isolation, processing, and coxl gene amplification}

Referring to Chambers et al. [34], microsatellite markers were isolated from the whole genome of Ae. albopictus by magnetic-bead enrichment and PCR screening, and all markers were found to be highly polymorphic via denatured polyacrylamide gel electrophoresis (D-PAGE). A set of 11 microsatellite loci was employed for genotyping the 17 Ae. albopictus populations. Detailed microsatellite primer information is listed in Table 1. All PCRs were performed on a T100 Thermal Cycler (Bio-Rad, USA) in a $50 \mu \mathrm{l}$ reaction system containing $10 \mathrm{ng}$ DNA, 0.25 U PrimeSTAR HS DNA polymerase $(10 \mathrm{pmol} / \mu \mathrm{l}$, TaKaRa, Japan), $6 \mu \mathrm{M}$ dNTPs (2.5 mM each, TaKaRa, Japan) and ca. $5 \mu \mathrm{M}$ of each primer, with the program set to 35 cycles of $95^{\circ} \mathrm{C}$ for $30 \mathrm{~s}, 57^{\circ} \mathrm{C}$ for $30 \mathrm{~s}$ and $72{ }^{\circ} \mathrm{C}$ for $1 \mathrm{~min}$ followed by a final elongation at $72{ }^{\circ} \mathrm{C}$ for $10 \mathrm{~min}$. All products were then checked with $2 \%$ agarose gel electrophoresis under UV light and run on a 3730XL DNA Genetic Analyzer (Applied Biosystems, USA).

As described by Bonacum et al. [35], coxI sequence polymorphism was investigated among 497 individuals (22-30 individuals per locality). Briefly, DNA amplification of a 550-bp fragment of coxI was performed on a T100 Thermal Cycler (Bio-Rad, USA) with the following primer set: 5'-GGAGGATTTGGAAATTGATTAGTT C-3' (F-coxI) and 5'-CCCGGTAAAATTAAAATATAA ACTTC-3' $(\mathrm{R}-\operatorname{cox} I)$ in a $50 \mu$ l reaction mix containing $10 \mu \mathrm{l}$ PCR reaction buffer (TaKaRa, Japan), $4 \mu \mathrm{l}$ dNTPs (2.5 mM each, TaKaRa, Japan), $1 \mu$ primers (10 pmol/ $\mu$, TaKaRa, Japan) and $0.5 \mu$ PrimeSTAR HS DNA polymerase (TaKaRa, Japan). The PCR amplification program was set as follows: pre-denaturation at $94{ }^{\circ} \mathrm{C}$ for $3 \mathrm{~min}$, followed by 35 cycles of denaturation at $94{ }^{\circ} \mathrm{C}$ for $30 \mathrm{~s}$, annealing at $54{ }^{\circ} \mathrm{C}$ for $45 \mathrm{~s}$ and elongation at $72{ }^{\circ} \mathrm{C}$ for 1 min, with a final elongation at $72{ }^{\circ} \mathrm{C}$ for $10 \mathrm{~min}$. All the
PCR products were detected and separated by $2 \%$ agarose gel electrophoresis. The target fragments for coxI were then cut from the gel under UV light and purified with the GenElute ${ }^{\mathrm{TM}}$ PCR Clean-Up Kit (NA1020, SigmaAldrich, USA). Each purified PCR product was then cloned into the $\mathrm{pCR}^{\mathrm{TM}} 2.1$ vector with a TA Cloning ${ }^{\mathrm{TM}} \mathrm{Kit}$ (K202040, Invitrogen, USA) and selected by bacteria liquid PCR with the T7 promoter primers. Finally, at least 20 positive clones for each PCR product were sequenced on both strands using an ABI 3730XL automatic sequencer (Applied Biosystems, USA).

\section{Population structure analyses, phylogenetic genotyping and migration analyses}

The microsatellite data were processed with GeneMapper v.4.0 (Applied Biosystems, USA). All markers were tested for polymorphism by determining their polymorphism information content (PIC) values via PIC-Calc 0.6 [43], and the null allele frequency of each locus was assessed with Micro-Checker version 2.2.3 [44]. Allele frequency indices, including $\mathrm{n}_{\mathrm{a}}$ and $\mathrm{n}_{\mathrm{e}}$, were assessed with Cervus version 3.0.7 [45]. The Ho, He and FIS values of all populations were calculated with Arlequin version 3.5.2.2 [46]. Departure from Hardy-Weinberg equilibrium (HWE) and heterozygosity deficiency were assessed via Bottleneck version 1.2.02 [47]. Additionally, STRUCTURE version 2.3.4 [48] and the $\Delta K$ method of Evanno et al. [49] were employed to calculate the optimal $K$ value; the parameters were set as follows: (1) $K$ ranged from 1 to 20 with ten iterations for each $K$ value; (2) an admixture model was chosen with a Markov chain Monte Carlo algorithm run for 100,000 iterations and 1,000,000 repetitions. The optimal $K$ value was calculated via Structure Harvester: http://taylor0.biology.ucla.edu/struc tureHarvester/ and depicted with Distribut version 1.1. The DAPC analysis was performed on the microsatellite

Table 1 Information of microsatellite primers used for genotyping all the 17 Ae. albopictus populations

\begin{tabular}{|c|c|c|c|c|c|c|c|}
\hline No. & $\begin{array}{l}\text { Primer } \\
\text { name }\end{array}$ & $\begin{array}{l}\text { Forward sequence } \\
\left(5^{\prime}-3^{\prime}\right)\end{array}$ & Reverse sequence $\left(5^{\prime}-3^{\prime}\right)$ & Repeat motifs & Size range (bp) & Dye & $\begin{array}{l}\text { GenBank } \\
\text { no. }\end{array}$ \\
\hline 1 & BW-P1 & TTAGCATCCATCTATTCTGGC & AAACATTCCTACGCATTTCAC & $(\mathrm{GT}) 6$ & $230-260$ & 5'-HEX & MT642446 \\
\hline 2 & BW-P3 & GAAAATATGGTCTATCAAATG & AAGTCAGTAAAACAGGAGTCT & $(\mathrm{GT}) 3 \mathrm{GC}(\mathrm{GT}) 3 \mathrm{TTT}$ & $132-178$ & $5^{\prime}-\mathrm{FAM}$ & MT642447 \\
\hline 3 & BW-P6 & GAATTGGGAGCTTGGTAAAAC & CGCCTACTTGAGAAACACTGA & $(\mathrm{TG}) 5$ & $124-200$ & 5'-HEX & MT642448 \\
\hline 4 & BW-P18 & CACTGGTTCTCTATCGAATGC & GTGTTATCAGTTGGAAGCGTT & $(\mathrm{GT}) 24$ & $158-203$ & $5^{\prime}-\mathrm{HEX}$ & MT642449 \\
\hline 5 & BW-P22 & GGCGTCCCCCCAACATACATC & CAGCTCCGTCCTCCTCTTCCC & $(\mathrm{GT}) 5(\mathrm{GCGT}) 2(\mathrm{GT}) 3$ & $187-244$ & 5'-HEX & MT642450 \\
\hline 6 & BW-P23 & GGATAAGAATGACACAGGCAC & CAAAGAGGAACACCATAGGAA & $(\mathrm{GAC}) 7$ & $133-177$ & $5^{\prime}-\mathrm{FAM}$ & MT642451 \\
\hline 7 & BW-P24 & ACGAAACATACTTACAATTGCA & AACCTAGAGTCCGAGAGAGAAC & $(\mathrm{AC}) 8$ & $145-239$ & $5^{\prime}$-FAM & MT642452 \\
\hline 8 & BW-P26 & CGTGGTGGTTAGGTCCATGTT & TCGCTTTCGGCTCTAGTCAAT & $(\mathrm{GT}) 5$ & $107-233$ & $5^{\prime}-\mathrm{HEX}$ & MT642453 \\
\hline 9 & BW-P27 & TTATACAAAAAGCGAACATCC & CACACACATAGAAAAAAGCAA & $(A C G) 6$ & $249-281$ & $5^{\prime}-\mathrm{FAM}$ & MT642454 \\
\hline 10 & BW-P35 & TATTTGCACATCCATTTCGTCT & TTCAAAACCTGATTTCCGACTG & (CA)7ТTТ & $83-120$ & 5'-HEX & MT642455 \\
\hline 11 & BW-P36 & GTCATGTAGTCCTCACAGTCAC & ATATGGATCATAGATGATGGAG & $(\mathrm{AC}) 6 \mathrm{CCC}$ & $159-179$ & $5^{\prime}-\mathrm{FAM}$ & MT642456 \\
\hline
\end{tabular}


date of each population with the $\mathrm{R}$ packages "Adegenet 2.1.3" [50], and the IBD analysis was performed between genetic distance, generated from the microsatellite date, and geographical distance with "Genpop 4.7.5" [51], respectively; meanwhile, the genetic variation and $\mathrm{F}_{\mathrm{ST}}$ value for each population were evaluated with the AMOVA test via Arlequin version 3.5.2.2 [46].

The haplotypes of all the populations were screened by DnaSP version 6.0 [52], and the genetic relationships among all the haplotypes were displayed as a TCS network constructed by Network 10.0.0.0 [53, 54]. Moreover, BEAST version 1.8.4 [55] and the R package "pheatmap" [56] were used to build a phylogenetic tree of all the haplotypes and examine the distribution of all the haplotypes among the different climatic regions, with the best model selected by JModelTest 2.1.10 [57]. The mismatch distribution and Bayesian skyline plot analyses were conducted with Arlequin version 3.5.2.2 and BEAST version 1.8.4, respectively, to investigate the current dispersal incidences of each population. Concurrently, the possible migration routes were rebuilt via the $\mathrm{R}$ package "divMigrate" with the number of bootstrap replicates set to 3 , the alpha value set to 0.05 , the $\mathrm{Nm}$ method chosen for the migration statistic and the filter threshold value set to 0.25 [58]. Finally, the relationships between the genetic indices (i.e., $n_{e}$, Shannon index, $\mathrm{Hd}, \mathrm{H}$ and $\pi$ ) and environmental factors (i.e., temperature, altitude and rainfall) were described via principal component analysis (PCA) and multiple factor analysis (MFA) with the $\mathrm{R}$ package "FactoMineR" [59].

\section{Results}

\section{Microsatellite marker isolation and assessment}

In the present study, a total of 44 pairs of microsatellite markers were isolated from the whole genome of $A e$. albopictus, 11 pairs of which were highly polymorphic and were therefore chosen for microsatellite genotyping analysis (Table 1). The allele number of each locus ranged from 10 to 33, with a mean of 17.545 alleles per locus. The PIC values of each locus ranged from 0.334 to 0.925 , with a mean value of 0.713 . According to the definition of PIC values by Allah et al. [60], nearly all the markers selected were highly informative (PIC value $>0.5$ ); the exception was BW-P18, which had a value of 0.357 and was considered to be an informative marker. The MicroChecker results suggested that null alleles were present at all loci, with frequencies ranging from 0.064 to 0.157 , with an average of 0.078 (Additional file 2: Table S5); however, frequency values $<0.2$ are considered to have no significant effect on the accuracy of data analysis by many studies [61, 62]. The linkage disequilibrium (LD) test showed that 302 pairs of loci of a total of 1870 (16.15\%) across all locations were in significant LD after
Bonferroni correction, while no consistency was found among them (Additional file 3: Figure S1).

\section{Genetic diversity and variation}

According to the results of the microsatellite data analysis, the observed number of alleles $\left(\mathrm{n}_{\mathrm{a}}\right)$ in each Ae. albopictus population was very high, and the mean $\mathrm{n}_{\mathrm{a}}$ value in each climatic region ranged from 6.909 to 8.091 without a significant difference. In contrast, the effective number of alleles $\left(n_{e}\right)$ ranged from 3.501 to 4.525 , and the $\mathrm{n}_{\mathrm{e}}$ value increased from the temperate region (3.876) to the tropical region (4.144). The mean value of observed heterozygosity (Ho) for all the climatic regions was ca. 0.557 , which was significantly lower than the expected heterozygosity (He) (ca. 0.684). The $\mathrm{F}_{\mathrm{IS}}$ value of each climatic region ranged from 0.266 to 0.359 , and the $A e$. albopictus populations significantly departed from HWE, except in two locations, SHJD and KZXZ (subtropical region, Table 2). Heterozygosity tests of all 17 Ae. albopictus populations based on the stepwise mutation model (SMM) revealed that nearly all the populations from the temperate and subtropical regions displayed significant population expansion with $p$ values $<0.05$, while no significance was observed among all the tests for the Ae. albopictus populations from the tropical region after Bonferroni correction (Additional file 4: Table S2).

In all, a total of 25 coxI haplotypes were observed among 497 Ae. albopictus individuals (GenBank ID: MN651301-MN651325) based on an analysis of the coxI sequences. The haplotype indices (i.e., $\mathrm{Hd}, \mathrm{H}$, and $\pi$ ) changed dramatically across Ae. albopictus populations from different climatic regions (Additional file 5: Table S3). The haplotype diversity (Hd) ranged from 0.074 (ZGND, temperate region) to 0.750 (JYJB, tropical region), with the nucleotide diversity $(\pi)$ ranging from 0.014 (BJLG, temperate region) to 0.190 (JYJB, tropical region). The average number of nucleotide differences (k) ranged from 0.067 (BJLG, temperate region) to 0.929 (JYJB, tropical region), and the number of polymorphic sites ranged from 1 to 5 across all the populations. Overall, the tropical Ae. albopictus populations showed the highest diversity, with mean values of $\mathrm{Hd}, \pi$ and $k$ reaching $0.657,0.156$ and 0.763 , respectively, whereas the temperate populations showed the lowest diversity.

\section{Population structure and differentiation based on microsatellite analysis}

In the present study, according to the microsatellite analysis results, all the Ae. albopictus populations were adequately allocated to two clades with significant genetic differences, and the best $K$ value, as determined via the $\Delta K$ method of Evanno et al., was also equal to two (Fig. 2a). Combined with the STRUCTURE bar plot 
Table 2 Genetic diversity indices for 17 Ae. albopictus populations investigated by 11 microsatellite loci

\begin{tabular}{|c|c|c|c|c|c|c|c|c|}
\hline Regions & SC & SS & $\mathbf{n}_{\mathrm{a}}$ & $n_{e}$ & $F_{\text {IS }}$ & Ho & $\mathrm{He}$ & HWE \\
\hline \multirow[t]{4}{*}{ Tropical region } & HKWN & 30 & 8.455 & 4.525 & 0.388 & 0.561 & 0.733 & $0.442^{* * *}$ \\
\hline & JYJB & 30 & 7.636 & 4.355 & 0.385 & 0.584 & 0.699 & $0.535^{* * *}$ \\
\hline & $\mathrm{JKCH}$ & 25 & 7.000 & 3.553 & 0.292 & 0.532 & 0.671 & $0.246^{* * *}$ \\
\hline & Mean & 29 & 7.697 & 4.144 & 0.355 & 0.559 & 0.701 & $0.408^{* * *}$ \\
\hline \multirow[t]{4}{*}{ South-subtropical region } & NNXD & 30 & 7.909 & 4.000 & 0.336 & 0.533 & 0.700 & $0.347^{* *}$ \\
\hline & NNXZ & 30 & 7.909 & 4.190 & 0.473 & 0.627 & 0.725 & $0.480^{* * *}$ \\
\hline & GZTH & 30 & 8.455 & 4.292 & 0.172 & 0.467 & 0.714 & $0.192^{*}$ \\
\hline & Mean & 30 & 8.091 & 4.161 & 0.327 & 0.542 & 0.713 & $0.340^{* *}$ \\
\hline \multirow[t]{5}{*}{ North-subtropical region } & NJTH & 30 & 7.455 & 3.836 & 0.392 & 0.590 & 0.682 & $0.473^{* * *}$ \\
\hline & NJDX & 30 & 8.455 & 4.119 & 0.465 & 0.637 & 0.706 & $0.551^{* * *}$ \\
\hline & $K Z X Z$ & 30 & 6.182 & 3.681 & 0.189 & 0.474 & 0.677 & 0.331 \\
\hline & SHJD & 30 & 5.455 & 3.106 & 0.035 & 0.455 & 0.606 & 0.123 \\
\hline & Mean & 30 & 6.909 & 3.677 & 0.266 & 0.542 & 0.660 & $0.369^{*}$ \\
\hline \multirow[t]{8}{*}{ Temperate region } & HNDX & 30 & 7.000 & 3.642 & 0.247 & 0.553 & 0.627 & $0.365^{* *}$ \\
\hline & QDDX & 30 & 7.909 & 3.921 & 0.361 & 0.570 & 0.664 & $0.380^{* *}$ \\
\hline & $\mathrm{BHBG}$ & 22 & 8.091 & 3.838 & 0.433 & 0.641 & 0.637 & $0.447^{* * *}$ \\
\hline & BJLG & 30 & 8.636 & 4.341 & 0.408 & 0.574 & 0.728 & $0.456^{* * *}$ \\
\hline & ZGND & 30 & 6.909 & 3.728 & 0.425 & 0.631 & 0.636 & $0.419^{* * *}$ \\
\hline & SXJW & 30 & 6.455 & 3.501 & 0.270 & 0.572 & 0.622 & $0.421^{* *}$ \\
\hline & HBSD & 30 & 7.546 & 3.926 & 0.256 & 0.508 & 0.687 & $0.341^{* *}$ \\
\hline & Mean & 29 & 7.591 & 3.876 & 0.359 & 0.583 & 0.662 & $0.411^{* *}$ \\
\hline
\end{tabular}

SC sample code, SS sample size, $n_{a}$ observed number of alleles, $n_{e}$ effective number of alleles (Kimura and Crow 1964), HWE Hardy-Weinberg disequilibrium, Ho observed heterozygosity, He expected heterozygosity (Nei's 1973)

${ }^{* * *} p<0.001$

${ }^{* *} p<0.01$

${ }^{*} p<0.05$

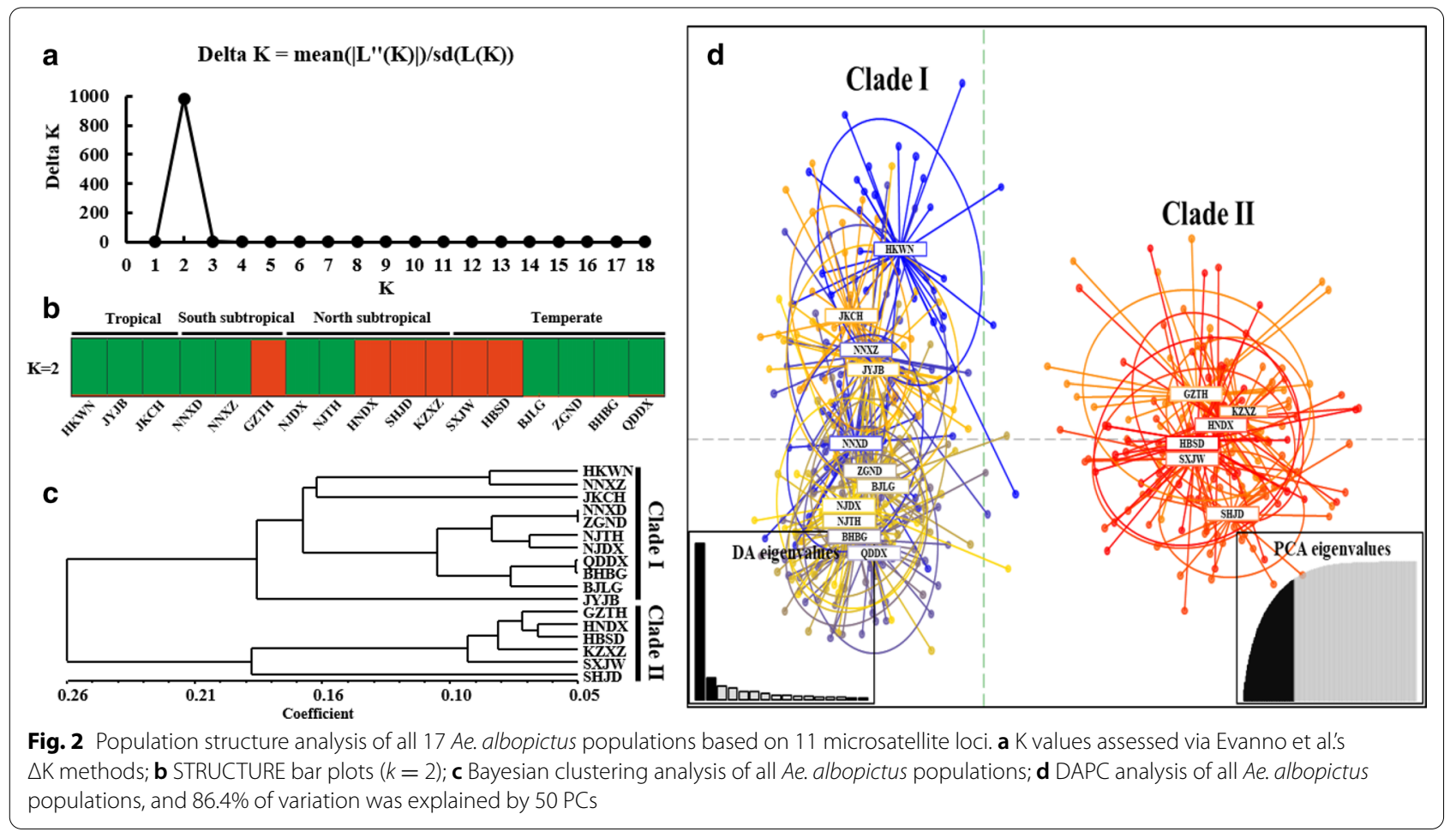


analysis, the Bayesian clustering analysis showed that all the Ae. albopictus populations were adequately allocated to two clades with certain locations from the subtropical and temperate regions genetically isolated from the other locations. (Fig. 2b and c). Moreover, a total of $86.4 \%$ of the variation was explained by 50 PCs in the discriminant analysis of principal components (DAPC) analysis; the results revealed two genetically isolated groups, and there was no clear relationship between the Ae. albopictus population structure and its distribution across climatic regions (Fig.2d).

The AMOVA results are presented in Table 3. Of the total genetic variation partitioned, $31.40 \%$ could be attributed to differences among individuals within populations and $63.04 \%$ to differences within individuals $\left(F_{\mathrm{IS}}=\right.$ $0.33253, F_{\mathrm{IT}}=0.36962$, and all $\left.p<0.00001\right)$. Meanwhile, the pairwise $F_{\mathrm{ST}}$ values between populations ranged from 0.008 (ZGND, temperate region and NNXD, subtropical region) to 0.141 (SHJD, subtropical region and JKCH, tropical region). Genetic differentiation was significant between all the sampled populations after Bonferroni correction $(p<0.05)$ except for five pairs of $\mathrm{F}_{\mathrm{ST}}$ values among the subtropical populations NNXZ, NJTH, NJDX, KZXZ and temperate populations ZGND, BHBG, QDDX and HBSD (Additional file 6: Table S4). In contrast to the high individual variation, a slightly significant positive correlation was observed among only the temperate populations via isolation by distance (IBD) analysis $\left(R^{2}=\right.$ $0.6614, p=0.048$ ), while no such evidence was observed in the other two regions (Additional file 7: Figure S2).

\section{Haplotype network and phylogenetic analysis based on coxl sequences}

Three major haplotype clades, distributed across the three central climatic regions and closely related to each other, were reconstructed via the TCS network with 497 coxI sequences. Haplotype I (H1) of Clade III was the most frequent haplotype and was distributed from the tropical to the temperate region with an increasing trend. Nearly all the other haplotypes derived from $\mathrm{H} 1$ with one or two mutations, with Clade I mainly distributed in the tropical region and Clade II mainly distributed in the southern subtropical region (Fig. 3b).

A phylogenetic tree of all 25 haplotype sequences, combined with a heatmap analysis, demonstrated that the 25 haplotypes were divided into three major well-supported clades. As expected, Clade I was separated from Clade II and Clade III with $100 \%$ bootstrap support and included haplotypes $\mathrm{H} 9, \mathrm{H} 10, \mathrm{H} 11, \mathrm{H} 12, \mathrm{H} 19$ and $\mathrm{H} 20$, which were observed in only the tropical region. In comparison, Clade II diverged from Clade III with lower bootstrap support $(70.74 \%)$ and included five haplotypes (H2-H3 and $\mathrm{H} 5-\mathrm{H} 7)$ that were distributed in only the southern subtropical region. Clade III was an admixture group containing all the remaining 14 haplotypes, 2 of which were observed in the tropical region and four in the climatic regions (Fig. 3a). Meanwhile, the Tajima's D and Fu's $\mathrm{F}$ values were negative, with no statistically significant $p$ values except in population JKCH (tropical region, Additional file 5: Table S3). Based on the coxI sequences, the mismatch analysis results showed that the Harpending raggedness indices for all three haplotype clades were relatively low (ranging from 0.1054 to $0.1812, p>0.05$ ), and unimodal mismatch distributions were observed among the different Ae. albopictus populations (Additional file 8: Figure S3).

\section{Migration and correlation analyses between genetic indices and environmental factors}

As illustrated in Fig. 4, all five genetic indices (i.e., $n_{e}$, Shannon index, $\mathrm{Hd}, H$ and $\pi$ ) and two environmental factors (i.e., temperature and rainfall) contributed equivalently to the first axis of the PCA, explaining up to $95.2 \%$ of the variation; the exception was the environmental factor latitude, which contributed more to the second axis, with a proportion of $17.5 \%$. Combining these results with hierarchical clustering via MFA, all 17 Ae. albopictus populations were clustered into three groups, of which cluster II and cluster III were closely related to each other. When environmental factors were regarded as the major influencing factors, the molecular diversity indices (i.e., $\mathrm{Hd}, k$ and $\pi$ ) of the populations in the tropical region

Table 3 Analysis of molecular variance (AMOVA) test of 17 Ae. albopictus populations sampled from three climatic regions.

\begin{tabular}{|c|c|c|c|c|c|c|}
\hline Source of variation & d.f. & Sum of squares & Variance components & $\begin{array}{l}\text { Percentage } \\
\text { of variation }\end{array}$ & P value & $\begin{array}{l}\text { Fixation } \\
\text { indices }\end{array}$ \\
\hline Among groups & 5 & 142.425 & $0.12789 \mathrm{Va}$ & 3.62 & $P<0.00001$ & $F_{C T}=0.03620$ \\
\hline Among populations within groups & 11 & 93.157 & $0.06845 \mathrm{Vb}$ & 1.94 & $P<0.00001$ & $F_{S C}=0.02010$ \\
\hline Among individuals within populations & 485 & 2156.367 & $1.10951 \mathrm{VC}$ & 31.40 & $P<0.00001$ & $F_{I S}=0.33253$ \\
\hline Within individuals & 502 & 1118.000 & $2.22709 \mathrm{Vd}$ & 63.04 & $P<0.00001$ & $F_{\mid T}=0.36962$ \\
\hline Total & 1003 & 3509.949 & 3.53294 & & & \\
\hline
\end{tabular}



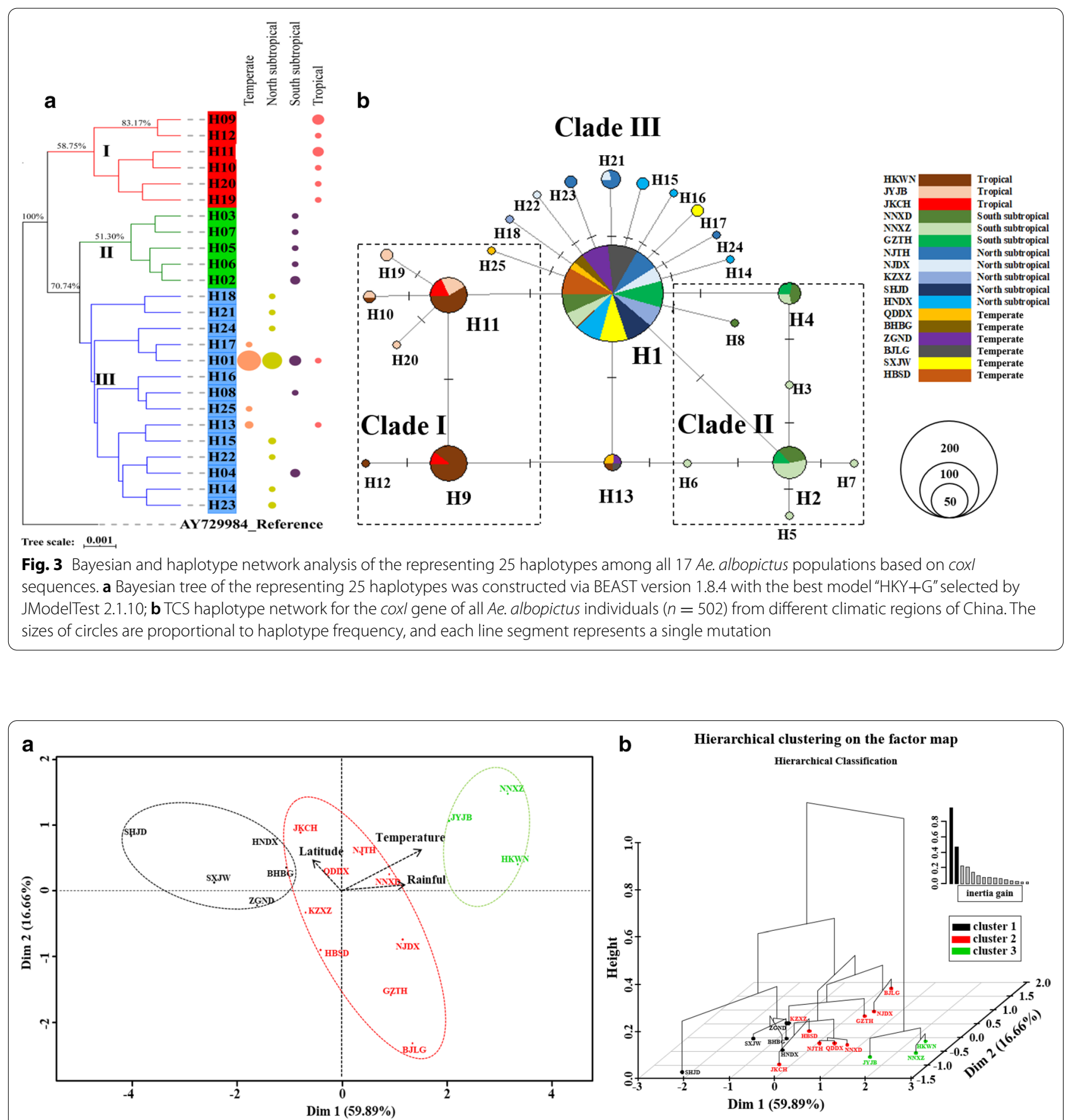

Fig. 4 Multiple factor analysis (MFA) and hierarchical clustering performed on 17 Ae. albopictus populations sampled from three different climatic regions. a Multiple factor analysis between genetic indices of all individuals and three environmental variables (temperature, rainfall and altitude) of 17 Ae. albopictus populations, populations of different clusters were marked with different colors; $\mathbf{b}$ hierarchical clustering of all 17 Ae. albopictus populations on the factor map

were significantly higher than those in the other regions (Additional file 5: Table S3).

Migration patterns were assessed using divMigrate networks representing all 17 Ae. albopictus populations. As expected, Ae. albopictus was observed to have migrated frequently between the tropical and temperate areas in both directions (Fig. 1). A total of four major migration trends were observed among the different climatic regions with high gene flow $(\mathrm{Nm}>0.4)$. Two trends were found to have originated from the tropical and 
subtropical areas and reached Hebei and Beijing in the temperate area, while another two arrived in Guangdong and Guangxi from the southern subtropical area. Compared with the south to north routes, the latter migration routes were substantially larger.

\section{Discussion}

Due to their high mutation rates, codominant expression and universal distribution throughout the eukaryotic genome, microsatellite loci have been widely used to evaluate the genetic variation and population structure of mosquitoes, especially for those without fully annotated genomes [32, 34]. Although different sets of microsatellite loci have been identified by previous studies [31, 32, 63], the absence of PCR amplification products of mosquito samples from certain climatic regions has resulted in insufficient data. Therefore, in this study, we developed new primers for our samples to ensure the integrity of the experimental data. However, the potential impact of null alleles, such as reducing population genetic diversity and increasing genetic differentiation among populations [61, $64,65]$, must be taken into consideration when microsatellite markers are used, because null alleles are more frequent in arthropods than in other species [66-68]. Mitochondrial genes are also good markers for studying population diversity and are widely used in many studies. To improve the accuracy of the findings in this study, the mitochondrial coxI gene was also employed, and the genetic variation of all the individuals was investigated using both coxI and microsatellite data.

Consistent with the studies of Zhong et al. [69] and Wei et al. [70], which were based on only mitochondrial and microsatellite diversity, respectively, the tropical Ae. albopictus populations showed higher diversity than the populations of the other two regions in China. An environment more conducive to survival and continuous dispersion may be the best explanation for this phenomenon. According to studies by Wei et al. and others [70-73], the hot and humid climate of Southern China is more suitable for the breeding and development of $A e$. albopictus than a temperate climate, which has relatively low temperatures and dry air. Notably, during the winter, freezing temperatures bring about diapause in Ae. albopictus for the whole winter period, resulting in lower allele richness and population diversity in temperate Ae. albopictus populations [66-68]. Meanwhile, Southern China shares borders with many countries in Southeast Asia, where Ae. albopictus originates geographically, including Laos, the Philippines, and Myanmar [23]. Frequent border trades and resident travel among these areas result in the continuous dispersion of Ae. albopictus, enriching the diversity of the local population; this result was confirmed by the results of the bottleneck analysis, which showed that all the populations from temperate and subtropical regions had undergone bottleneck effects, while the populations of the tropical region had not. As suggested by Takezaki et al. [74], an He value between 0.5 and 0.8 indicates high genetic polymorphism in the population. In the present study, the He values of all the sampling sites ranged from 0.606 to 0.733 , which indicates high polymorphism. Simultaneously, compared with those in the tropical region, the mean Ho values of the temperate and subtropical regions were significantly lower than the mean He values, and nearly all the populations significantly departed from HWE. This phenomenon may also be closely related to the less suitable climate and the need to develop diapause during wintertime among populations of these regions as mentioned before, and our present sampling method (larvae rather than adults) may also be the reason for the significant departure from HWE of all populations. Meanwhile, the continuous dispersion of Ae. albopictus from Southeast Asian countries to the tropical region of China also augments the local mosquito population (Additional file 9: Figure S4).

In the present study, nearly $44 \%$ (11 out of 25 ) of these 25 detected haplotypes were observed only in the tropical and subtropical regions. The same result for Chinese Ae. albopictus populations was also observed by both Guo et al. and Zhang et al. [16, 75]. Guo et al. identified three predominant haplotypes in Ae. albopictus populations: H01 (21.5\%) from Guangdong Province (subtropical region), H19 (22.1\%) from Yunnan (tropical region) and Hainan provinces (tropical region), and H30 (10\%) from Hainan Province (tropical region); likewise, Zhang et al. also detected 25 haplotypes across China, and $48 \%$ (12 out of 25 ) of these haplotypes were detected in only tropical and subtropical regions. Both studies corroborate our findings that the haplotype diversity of the tropical and subtropical regions is higher than that of the temperate region. Continuous dispersal from neighboring Southeast Asian countries may be the reason for the higher diversity of the Ae. albopictus populations in the tropical and subtropical regions and that the origins of the Ae. albopictus populations of these two regions may be different. Furthermore, the mismatch analysis results revealed recent population dispersal among the different climatic regions, which is the main reason for the universal distribution of the remaining haplotypes. As an important and rapidly adapting insect, Ae. albopictus has biological characteristics that are strongly influenced by environmental conditions $[9,10]$. In tropical and subtropical regions, the climate is hot and wet, making it more suitable for the survival of Ae. albopictus; when this mosquito migrates from a tropical region to a temperate region, it needs time to adapt to the new environment, 
especially to survive during the winter period, which causes diapause in Ae. albopictus [4]. According to a study by Roiz et al. about the effects of environmental factors on Ae. albopictus, the activity of host-seeking females was positively affected by temperature and rainfall [76]. The PCA in the present study examined genetic indices and environmental factors (such as temperature, rainfall and altitude) and revealed that temperature and rainfall may be the leading causes of differences in genetic diversity among Ae. albopictus populations from different climatic regions.

Corresponding to the results of Kotsakiozi and Manni et al. [32, 77], which showed no genetic differentiation within the native Asian range, our population differentiation analysis results showed that even though nearly all the pairwise $F_{\mathrm{ST}}$ values between populations were significant, the values were not high, which indicates potential anthropogenic exchange between the populations. A study by Schmidt et al. [78] of the genetic structure of $A e$. albopictus collected from 12 sample sites in Guangzhou also found that human transportation networks, particularly shipping terminals, influenced the genetic structure of Ae. albopictus populations. With the development of China, the rapid expansion of high-speed rail, aircraft routes and highways between different climatic regions has not only facilitated trade among different cities but also accelerated the spread of mosquitoes. Accordingly, only two genetically separated clades were observed among these populations, and molecular variations within populations and individuals were found to contribute to the genetic differentiation between these populations. As a global invasive species, Ae. albopictus has developed several strategies to cope with a broad range of temperatures and adapt to local thermal conditions [9, 71], which helps this mosquito disperse and colonize different locations successfully. Here, the migration analysis results displayed four major migration trends with high gene flow $(\mathrm{Nm}>0.4)$ among the different climatic regions, corresponding with China's main trade routes (e.g. pan-Pearl River Delta and Bohai Rim Business District, etc.) and tourism routes (e.g. Beijing-Guangzhou railway, Beijing-Shanghai railway and Beijing-Kowloon railway). All these results revealed that human-aided dispersion might be the main reason for the similarity of populations across the different regions [25, 70-74, 77-83]. This hypothesis was also verified via IBD analysis, which indicated that long distances were not significantly associated with individual genetic variation, except among individuals from the tropical region with a significant $p$ value of the Mantel test.

Overall, strong dispersal patterns were observed in Southern China, and frequent dispersals have occurred from the tropical and subtropical regions to the temperate region. Monitoring the population structure and potential migration routes of Ae. albopictus in different climatic regions will help in the selection of appropriate mosquito control strategies, and since dengue fever continues to break out in Southern China every year, monitoring the potential migration routes of Ae. albopictus can also predict the potential transmission routes of this virus and help prevent its further spread.

\section{Conclusion}

The present study systematically evaluated the genetic variation, population structure and haplotype-based phylogenetic relationships of Ae. albopictus populations across different climatic regions of China. All 17 Ae. albopictus populations were genetically structured into two isolated clades, in accordance with their locality or region of origin, and three major haplotype clusters were observed via phylogenetic analysis of coxI. These results suggest different evolutionary histories in changing environments, especially differences in temperature and rainfall. Meanwhile, four major migration trends were observed among the different climatic regions with high gene flow; these trends contribute to the similarity of the Ae. albopictus populations.

\section{Supplementary Information}

The online version contains supplementary material available at https://doi. org/10.1186/s13071-020-04521-4.

Additional file 1: Table S1. Sampling information of 17 Ae. albopictus populations collected from three different climatic regions of China.

Additional file 2: Table S5. Genetic diversity of 11 microsatellite loci developed for Ae. albopictus based on samples $(n=502)$ collected from three different climatic regions of China.

Additional file 3: Figure S1. Linkage disequilibrium analysis at each pair of Loci across all 17 Ae. albopictus populations.

Additional file 4: Table S2. Heterozygosity tests of all 17 Ae. albopictus populations based on SMM model.

Additional file 5: Table S3. Haplotype diversity of 17 Ae. albopictus populations based on cox/ collected from three different climatic regions of China.

Additional file 6: Table S4. Population differentiation estimation of the $\mathrm{F}_{\text {ST }}$ value (below the diagonal) and Geographic distance (above the diagonal) among all 17 Ae. albopictus populations.

Additional file 7: Figure S2. Isolation by distance analysis of all $17 \mathrm{Ae}$. albopictus populations.

Additional file 8: Figure S3. Historical demography analysis of Ae. albopictus inferred from mtDNA cox/ sequences.

Additional file 9: Figure S4. Migration analysis of all 17 Ae. albopictus populations inferred from microsatellite data.

\section{Abbreviations}

coxl: Mitochondrial cytochrome c oxidase subunit 1; PIC: Polymorphism Information Content; Ho: Observed heterozygosity; He: Excepted heterozygosity; HWE test: Hardy-Weinberg equilibrium test; AMOVA test: Analysis of molecular variation test; IBD: Isolation by distance; WHO: World Health Organization; CDC: Centers for Disease Control; $n_{a}$ : Observed number of alleles; $n_{e}$ : Effective 
number of alleles; $F_{15}$ : Inbreeding coefficient; $F_{S T}$ : Genetic differences among populations; $F_{1 T}$ : Genetic differences within individuals; PCA: Principal component analysis; MFA: Multiple factor analysis; LD: Linkage disequilibrium; $\mathrm{Hd}$ : Haplotype diversity; DAPC: Discriminant analysis of principal components; $\mathrm{Nm}$ : Number of migrants.

\section{Acknowledgements}

Not applicable.

\section{Authors' contributions}

$J G, H D Z, C X L$ and TYZ jointly designed and coordinated the study, with contributions from XXG, DX and YDD. JG drafted the article with contributions from HDZ. HDZ, JG, CJL and GW collected samples from different climatic regions of China. JG, HDZ, GW and CJL carried out the laboratory work and performed the statistical analysis. All authors read and approved the final manuscript.

\section{Funding}

This work was funded by grants from the Infective Diseases Prevention and Cure Project of China (No. 2017ZX10303404)

\section{Availability of data and material}

Data are available on request to the corresponding author.

\section{Ethics approval and consent to participate}

Not applicable.

\section{Consent for publication}

Not applicable.

\section{Competing interests}

The authors declare that they have no competing interests.

Received: 28 June 2020 Accepted: 4 December 2020

Published online: 06 January 2021

\section{References}

1. Cancrini G, Frangipane di A, Ricci I, Tessarin C, Gabrielli S, Pietrobelli M. Aedes albopictus is a natural vector of Dirofilaria immitis in Italy. Vet Parasitol. 2003;118:195-202.

2. Wong PS, Li MZ, Chong CS, Ng LC, Tan CH. Aedes (Stegomyia) albopictus (Skuse): a potential vector of Zika virus in Singapore. PLoS Negl Trop Dis. 2013;7:e2348.

3. Grard G, Caron M, Mombo IM, Nkoghe D, Mboui Ondo S, Jiolle D, et al. Zika virus in Gabon (Central Africa)-2007: a new threat from Aedes albopictus? PLoS Negl Trop Dis. 2014;8:e2681.

4. Kress A, Kuch U, Oehlmann J, Muller R. Effects of diapause and cold acclimation on egg ultrastructure: new insights into the cold hardiness mechanisms of the Asian tiger mosquito Aedes (Stegomyia) albopictus. J Vector Ecol. 2016;41:142-50.

5. Rezza G. Aedes albopictus and the reemergence of Dengue. BMC Public Health. 2012;12:72.

6. Kraemer MU, Sinka ME, Duda KA, Mylne AQ, Shearer FM, Barker CM, et al. The global distribution of the arbovirus vectors Aedes aegypti and Ae. albopictus. Elife. 2015;4:e08347.

7. Bonizzoni M, Gasperi G, Chen X, James AA. The invasive mosquito species Aedes albopictus: current knowledge and future perspectives. Trends Parasitol. 2013;29:460-8.

8. Ayres JS, Schneider DS. The role of anorexia in resistance and tolerance to infections in Drosophila. PLoS Biol. 2009;7:e1000150.

9. Culbert NJ, Gilles JRL, Bouyer J. Investigating the impact of chilling temperature on male Aedes aegypti and Aedes albopictus survival. PLoS ONE. 2019;14:e0221822.

10. Kweka E, Baraka V, Mathias L, Mwang'onde B, Baraka G, Lyaruu L, et al. Ecology of Aedes mosquitoes, the Major vectors of arboviruses in human population. In: Falcón-Lezama JA, Betancourt-Cravioto M, Tapia-Conyer $\mathrm{R}$, editors., et al., Dengue fever-a resilient threat in the face of innovation. London: IntechOpen; 2019. p. 40-50.
11. Catherine J, Westbrook MHR, Pesko KN, Greene KE, Lounibos LP. Larval environmental temperature and the susceptibility of Aedes albopictus Skuse (Diptera: Culicidae) to Chikungunya virus. Vector Borne Zoonotic Dis. 2010;10:241-7.

12. Ephantus J, Muturi MBJ, Montgomery A. Temperature and densitydependent effects of larval environment on Aedes aegypti competence for an alphavirus. J Vector Ecol. 2012;37:154-61.

13. Murdock CC, Evans MV, McClanahan TD, Miazgowicz KL, Tesla B. Finescale variation in microclimate across an urban landscape shapes variation in mosquito population dynamics and the potential of Aedes albopictus to transmit arboviral disease. PLoS Negl Trop Dis. 2017;11:e0005640.

14. Costanzo KS, Westby KM, Medley KA. Genetic and environmental influences on the size-fecundity relationship in Aedes albopictus (Diptera: Culicidae): Impacts on population growth estimates? PLOS ONE. 2018;13:e0201465.

15. Lubinda J, Trevino CJ, Walsh MR, Moore AJ, Hanafi-Bojd AA, Akgun S, et al. Environmental suitability for Aedes aegypti and Aedes albopictus and the spatial distribution of major arboviral infections in Mexico. Parasite Epidemiol Control. 2019;6:e00116.

16. Guo Y, Song Z, Luo L, Wang Q, Zhou G, Yang D, et al. Molecular evidence for new sympatric cryptic species of Aedes albopictus (Diptera: Culicidae) in China: a new threat from Aedes albopictus subgroup? Parasit Vectors. 2018;11:228.

17. Latreille AC, Milesi P, Magalon H, et al. High genetic diversity but no geographical structure of Aedes albopictus populations in Réunion Island. Parasites Vectors. 2019;12:597.

18. Lee EJ, Yang S-C, Kim T-K, et al. Geographical genetic variation and sources of Korean Aedes albopictus (Diptera: Culicidae) populations. J Med Entomol. 2020;57:1057-68.

19. Chadee DD, Martinez R. Aedes aegypti (L.) in Latin American and Caribbean region: with growing evidence for vector adaptation to climate change? Acta Trop. 2016;156:137-43.

20. Reba M, Reitsma F, Seto KC. Spatializing 6,000 years of global urbanization from 3700 BC to AD 2000. Sci Data. 2016;3:160034.

21. Jia P, Chen X, Chen J, Lu L, Liu Q, Tan X. How does the dengue vector mosquito Aedes albopictus respond to global warming? Parasit Vectors. 2017;10:140.

22. Johnson MTJ, Munshi-South J. Evolution of life in urban environments. Science. 2017;358:6363.

23. Lai S, Johansson MA, Yin W, Wardrop NA, van Panhuis WG, Wesolowski A, et al. Seasonal and interannual risks of dengue introduction from SouthEast Asia into China, 2005-2015. PLoS Negl Trop Dis. 2018;12:e0006743.

24. Xiang B, Gao P, Kang Y, Ren T. Importation of Zika virus in China: a significant risk in southern China. J Infect. 2017;74:328-30.

25. Wu F, Liu Q, Lu L, Wang J, Song X, Ren D. Distribution of Aedes albopictus (Diptera: Culicidae) in northwestern China. Vector Borne Zoonotic Dis. 2011;11:1181-6.

26. Zheng $X$, Zhong D, He Y, Zhou G. Seasonality modeling of the distribution of Aedes albopictus in China based on climatic and environmental suitability. Infect Dis Poverty. 2019;8:98.

27. Wu JY, Lun ZR, James AA, Chen XG. Dengue fever in mainland China. Am J Trop Med Hyg. 2010;83:664-71.

28. Lei Luo LYJ, Yang ZC. The dengue preface to endemic in mainland China: the historical largest outbreak by Aedes albopictus in Guangzhou, 2014. Infect Dis Poverty. 2017;6:148.

29. Powell JR. Genetic variation in insect vectors: death of typology? Insects. 2018;9:139.

30. Vieira ML, Santini L, Diniz AL, Munhoz CF. Microsatellite markers: what they mean and why they are so useful. Genet Mol Biol. 2016;39:312-28.

31. Porretta D, Gargani M, Bellini R, Calvitti M, Urbanelli S. Isolation of microsatellite markers in the tiger mosquito Aedes albopictus (Skuse). Mol Ecol Notes. 2006;6:880-1.

32. Manni M, Gomulski LM, Aketarawong N, Tait G, Scolari F, Somboon P, et al. Molecular markers for analyses of intraspecific genetic diversity in the Asian Tiger mosquito, Aedes albopictus. Parasit Vectors. 2015;8:188.

33. Multini LC, Marrelli MT, Wilke AB. Microsatellite loci cross-species transferability in Aedes fluviatilis (Diptera:Culicidae): a cost-effective approach for population genetics studies. Parasit Vectors. 2015;8:635.

34. Chambers EW, Meece JK, McGowan JA, Lovin DD, Hemme RR, Chadee $\mathrm{DD}$, et al. Microsatellite isolation and linkage group identification in the yellow fever mosquito Aedes aegypti. J Hered. 2007;98:202-10. 
35. Bonacum J, DeSalle R, O'Grady P, Olivera D, Wintermute J, Zilversmit M. New nuclear and mitochondrial primers for systematics and comparative genomics in Drosophilidae. Dros Inf Serv. 2001;84:201-4.

36. Park DS, Suh SJ, Hebert PD, Oh HW, Hong KJ. DNA barcodes for two scale insect families, mealybugs (Hemiptera: Pseudococcidae) and armored scales (Hemiptera: Diaspididae). Bull Entomol Res. 2011;101:429-34.

37. Shaikevich E, Talbalaghi A. Molecular Characterization of the Asian Tiger Mosquito Aedes albopictus (Skuse) (Diptera: Culicidae) in Northern Italy. ISRN Entomol. 2013;2013:1-6.

38. Hernández-Triana LM, Brugman VA, Nikolova NI, Ruiz-Arrondo I, Barrero E, Thorne $L$, et al. DNA barcoding of British mosquitoes (Diptera, Culicidae) to support species identification, discovery of cryptic genetic diversity and monitoring invasive species. Zookeys. 2019;832:57-76.

39. Sun F. Achieving suitable thermal performance in residential buildings in different climatic regions of China. Energy Build. 2013;67:11-21.

40. Kang L, Zhang H. A Comprehensive Study of Agricultural Drought Resistance and Background Drought Levels in Five Main Grain-Producing Regions of China. Sustainability. 2016;8:1-12.

41. Lu B, Wu H. Classification and identification of important medical insects of China. 1st ed. Henan: Henan Science and Technology Publishing House; 2003.

42. Norbert Becker ADP. Mosquitoes: identification, ecology and control. 3rd ed. New York: Springer International Publishing; 2019.

43. Nagy S, Poczai P, Cernak I, Gorji AM, Hegedus G, Taller J. PICcalc: an online program to calculate polymorphic information content for molecular genetic studies. Biochem Genet. 2012;50:670-2.

44. Van Oosterhout C, Hutchinson WF, Wills DPM, Shipley P. micro-checker: software for identifying and correcting genotyping errors in microsatellite data. Mol Ecol Notes. 2004;4:535-8.

45. Zhang H-R, Niu S-F, Wu R-X, Zhai Y, Tian L-T. Development and characterization of 26 polymorphic microsatellite markers in Lateolabrax maculatus and cross-species amplification for the phylogenetically related taxa. Biochem Syst Ecol. 2016;66:326-30.

46. Excoffier LLH. An integrated software package for population genetics data analysis. Evol Bioinform Online. 2015;1:47-50.

47. Cristescu R, Sherwin WB, Handasyde K, Cahill V, Cooper DW. Detecting bottlenecks using BOTTLENECK 1.2.02 in wild populations: the importance of the microsatellite structure. Conserv Genet. 2009;11:1043-9.

48. Earl DA, vonHoldt BM. STRUCTURE HARVESTER: a website and program for visualizing STRUCTURE output and implementing the Evanno method. Conserv Genet Res. 2011:4:359-61.

49. Evanno G, Regnaut S, Goudet J. Detecting the number of clusters of individuals using the software STRUCTURE: a simulation study. Mol Ecol. 2005;14(8):2611-20.

50. Jombart T. adegenet: a R package for the multivariate analysis of genetic markers. Bioinformatics. 2008;24:1403-5.

51. Mahesh BA, Kannan E, Davis GDJ, Venkatesan P, Ragunath PK. GenPop-An online tool to analyze human population genetic data. Bioinformation. 2020;16:149-52.

52. Rozas J, Sanchez-DelBarrio JC, Messeguer X, Rozas R. DnaSP, DNA polymorphism analyses by the coalescent and other methods. Bioinformatics. 2003;19:2496-7.

53. Frohlich F, Schmidt SL. Rational design of transcranial current stimulation (TCS) through mechanistic insights into cortical network dynamics. Front Hum Neurosci. 2013;7:804

54. Murias dos Santos A, Cabezas MP, Tavares Al, Xavier R, Branco M. tcsBU: a tool to extend TCS network layout and visualization. Bioinformatics. 2016;32:627-8

55. Drummond AJ, Suchard MA, Xie D, Rambaut A. Bayesian phylogenetics with BEAUti and the BEAST. Mol Biol Evol. 2012;29:1969-73.

56. Zhao S, Guo Y, Sheng Q, et al. Heatmap3: an improved heatmap package with more powerful and convenient features. BMC Bioinform. 2014;15:16.

57. Guindon S, Gascuel O. A simple, fast, and accurate algorithm to estimate large phylogenies by maximum likelihood. Syst Biol. 2003;52:696-704

58. Kharzinova VR, Dotsev AV, Kramarenko AS, Layshev KA, Romanenko TM, Solov'eva AD, et al. Study of the allele pool and the degree of genetic introgression of semi-demesticated and wild populations of reindeer (Rangifer tarandus L., 1758) using microsatellites. Sel'skokhozyaistvennaya Biologiya. 2016;51:811-23.

59. Lê Sébastien JJ, Husson F. FactoMineR: an R Package for Multivariate Analysis. J Stat Softw. 2008;25:1-18.
60. Ditta A, Zhou Z, Cai X, Shehzad M, Wang X, Okubazghi K, et al. Genomewide mining and characterization of SSR markers for gene mapping and gene diversity in Gossypium barbadense L. and Gossypium darwinii G. Watt Accessions. Agronomy. 2018;8:1-12.

61. Chapuis MP, Estoup A. Microsatellite null alleles and estimation of population differentiation. Mol Biol Evol. 2007;24:621-31.

62. Dakin EE, Avise JC. Microsatellite null alleles in parentage analysis. Heredity. 2004;93:504-9.

63. Delatte H, Toty C, Boyer S, Bouetard A, Bastien F, Fontenille D. Evidence of habitat structuring Aedes albopictus populations in Reunion Island. PLoS Negl Trop Dis. 2013;7:e211.

64. Girard P. A robust statistical method to detect null alleles in microsatellite and SNP datasets in both panmictic and inbred populations. Stat Appl Genet Mol Biol. 2011;10:artica19.

65. Rico C, Cuesta JA, Drake P, Macpherson E, Bernatchez L, Marie AD. Null alleles are ubiquitous at microsatellite loci in the Wedge Clam (Donax trunculus). PeerJ. 2017;5:e3188.

66. Lehmann T, Besansky NJ, Hawley WA, Fahey TG, Kamau L, Collins FH. Microgeographic structure of Anopheles gambiae in western Kenya based on mtDNA and microsatellite loci. Mol Ecol. 1997;6:243-53.

67. Meglecz E, Petenian F, Danchin E, D'Acier AC, Rasplus JY, Faure E. High similarity between flanking regions of different microsatellites detected within each of two species of Lepidoptera: Parnassius apollo and Euphydryas aurinia. Mol Ecol. 2004;13:1693-700.

68. Chapuis M-P, Loiseau A, Michalakis Y, Lecoq M, Estoup A. Characterization and PCR multiplexing of polymorphic microsatellite loci for the locust Locusta migratoria. Mol Ecol Notes. 2005;5:554-7.

69. Zhong D, Lo E, Hu R, Metzger ME, Cummings R, Bonizzoni M, et al. Genetic analysis of invasive Aedes albopictus populations in Los Angeles County, California and its potential public health impact. PLoS ONE. 2013;8:e68586.

70. Wei Y, Wang J, Song Z, He Y, Zheng Z, Fan P, et al. Patterns of spatial genetic structures in Aedes albopictus (Diptera: Culicidae) populations in China. Parasit Vectors. 2019;12:552.

71. Marinho RA, Bezerra-Gusmão MA, Porto VS, Olinda RA, dos Santos CAC. Effects of temperature on the life cycle, expansion, and dispersion of Aedes aegypti (Diptera: Culicidae) in three cities in Paraiba, Brazil. J Vector Ecol. 2016;41:10.

72. Mogi M, Armbruster PA, Tuno N, Aranda C, Yong HS. The climate range expansion of Aedes albopictus (Diptera: Culicidae) in Asia inferred from the distribution of Albopictus Subgroup Species of Aedes (Stegomyia). J Med Entomol. 2017:54:1615-25.

73. Jia P, Liang L, Tan X, Chen J, Chen X. Potential effects of heat waves on the population dynamics of the dengue mosquito Aedes albopictus. PLoS Negl Trop Dis. 2019;13:e0007528.

74. Naoko Takezaki AMN. Genetic distances and reconstruction of phylogenetic trees from microsatellite DNA. Genetics. 1996;144:389-99.

75. Ruiling Z, Peien L, Xuejun W, Zhong Z. Molecular analysis and genetic diversity of Aedes albopictus (Diptera, Culicidae) from China. Mitochondrial DNA A DNA Mapp Seq Anal. 2018;29:594-9.

76. David Roiz RR, Arnoldi D, Rizzoli A. Effects of temperature and rainfall on the activity and dynamics of host-seeking Aedes albopictus females in Northern Italy. Vector Borne Zoonotic Dis. 2010;10:811-6.

77. Kotsakiozi P, Richardson JB, Pichler V, Favia G, Martins AJ, Urbanelli S, et al. Population genomics of the Asian tiger mosquito, Aedes albopictus: insights into the recent worldwide invasion. Ecol Evol. 2017;7:10143-57.

78. Schmidt TL, Ri G, Zhang D, Zheng X, Xi Z, Hoffmann AA. Genome-wide SNPs reveal the drivers of gene flow in an urban population of the Asian Tiger mosquito, Aedes albopictus. PLOS Negl Trop Dis. 2017;11:e0006009.

79. Wondji C, Simard F, Lehmann T, Fondjo E, Same-Ekobo A, Fontenille D. Impact of insecticide-treated bed nets implementation on the genetic structure of Anopheles arabiensis in an area of irrigated rice fields in the Sahelian region of Cameroon. Mol Ecol. 2005;14:3683-9.

80. Chen H, Li K, Wang X, Yang X, Lin Y, Cai F, et al. First identification of $k d r$ allele F1534S in VGSC gene and its association with resistance to pyrethroid insecticides in Aedes albopictus populations from Haikou City, Hainan Island, China. Infect Dis Poverty. 2016;5:31.

81. Li Y, Xu J, Zhong D, Zhang H, Yang W, Zhou G, et al. Evidence for multipleinsecticide resistance in urban Aedes albopictus populations in southern China. Parasit Vectors. 2018;11:4 
82. Su X, Guo Y, Deng J, Xu J, Zhou G, Zhou T, et al. Fast emerging insecticide resistance in Aedes albopictus in Guangzhou, China: alarm to the dengue epidemic. PLoS Negl Trop Dis. 2019;13:e0007665.

83. Liu H, Liu L, Cheng P, Yang L, Chen J, Lu Y, et al. Bionomics and insecticide resistance of Aedes albopictus in Shandong, a high latitude and high-risk dengue transmission area in China. Parasit Vectors. 2020;13:11.

\section{Publisher's Note}

Springer Nature remains neutral with regard to jurisdictional claims in published maps and institutional affiliations.
Ready to submit your research? Choose BMC and benefit from:

- fast, convenient online submission

- thorough peer review by experienced researchers in your field

- rapid publication on acceptance

- support for research data, including large and complex data types

- gold Open Access which fosters wider collaboration and increased citations

- maximum visibility for your research: over $100 \mathrm{M}$ website views per year

At BMC, research is always in progress.

Learn more biomedcentral.com/submissions 